\title{
RELATION BETWEEN SERUM FERRITIN AND LEFT VENTRICULAR FUNCTIONS IN HEMODIALYSIS PATIENTS
}

\author{
By \\ Ahmed Lotfy Ahmed ${ }^{\text {a }}$, Fawzy Hamed Hassana, El-Sayed Mohamed \\ Rashed $^{\mathrm{a}}$, Mohamed Ahmed Mosaad ${ }^{\mathrm{b}}$ and Nabil Fathy Esmaeel ${ }^{\mathrm{c}}$ \\ a Department of Internal Medicine, Faculty of Medicine, Al Azhar University \\ ${ }^{b}$ Department of Cardiology, Faculty of Medicine, Al Azhar University \\ ${ }^{c}$ Department of Clinical pathology, Faculty of Medicine, Al Azhar University \\ E-mail: lotfiahmed272@gmail.com
}

\begin{abstract}
Background: Cardiovascular disease is still the most common cause of morbidity and mortality in hemodialysis patients. High serum ferritin is correlated with mortality and cardiovascular outcome in maintenance hemodialysis patients.

Objective: To evaluate relationship between serum ferritin level and left ventricular functions and cardiovascular outcomes in hemodialysis patients.

Patients and methods: Sixty hemodialysis (HD) patients from Clinic of Internal Medicine Department, AlSayed Galal University Hospital, Cairo, Egypt between December 2019 and June 2020. Were enrolled Left ventricular mass (LVM), Left ventricular mass index (LVMi) and Left ventricular mass/height 2.7 (LVM/Ht2.7) were evaluated with transthoracic echocardiography. Patients were classified into two groups according to serum ferritin into 30 patients with serum Ferritin $<800 \mathrm{ng} / \mathrm{ml}$ (Group A), and 30 patients with serum Ferritin $\geq 800 \mathrm{ng} / \mathrm{ml}$ (Group B).

Results: We found statistically significant difference ( $\mathrm{p}$-value $<0.05$ ) between studied groups as regard LVESD and LVM/Ht2.7, and statistical significant difference ( $\mathrm{p}$-value $<0.001)$ between studied groups as regard LVEDD and LVMI. The LVEDD, LVMI, LVESD and LVM/Ht2.7 values were significantly higher in group B (High Ferritin) compared with group A (Low ferritin).
\end{abstract}

Conclusion: High serum ferritin level $(\geq 800 \mu \mathrm{g} / \mathrm{L}$ ) was positively associated with left ventricular hypertrophy in hemodialysis patients.

Key Words: Left Ventricular Mass Index, Ferritin, Hemodialysis.

\section{INTRODUCTION}

Chronic kidney disease (CKD) is a global public health problem with a rising prevalence. Low glomerular filtration rate is associated with higher risk for kidney failure requiring dialysis, as well as with cardiovascular disease (CVD), hypertension, anemia, and other metabolic complications (Abdel-Hady et al., 2013). Cardiovascular disease is still the most common cause of morbidity and mortality in hemodialysis patients (Monfared et al., 2013). Left ventricular hypertrophy $(\mathrm{LVH})$ is frequent in maintenance hemodialysis (HD) patients and associated with a poor outcome. The worsening of preexisting $\mathrm{LVH}$ is the strongest predictor 
of sudden cardiac death in dialysis patients (Xu et al., 2013). Serum ferritin is widely recognized as an acute phase reactant that is nonspecifically enhanced under systemic inflammatory conditions, including chronic kidney disease (CKD), liver disease, and cancer (Khanna et al., 2017). Higher serum ferritin levels can induce macrophage accumulation and increase reactive oxygen species (ROS) formation during inflammation ( $F u$ et al., 2020). It was recently noted that serum ferritin concentration is highly correlated with mortality and cardiovascular outcome in maintenance hemodialysis patients (Lien et al., 2015).

The goal of our study was to evaluate the relationship between serum ferritin and left ventricular functions and cardiovascular outcomes in hemodialysis patients.

\section{PATIENTS AND METHODS}

The study was carried out on Internal Medicine Department, Al-Sayed Galal University Hospital, Cairo, Egypt, between December 2019 and June 2020. Sixty hemodialysis (HD) patients were enrolled.

Inclusion criteria: Adult patients aged 18 years or more with ESRD on maintenance HD for 3 months or more.

Exclusion criteria: Patients with advanced cardiac diseases, Patients who have malignant diseases, Patients with severe hepatic impairment, and patients who were treated with statins and/or nonsteroidal anti-inflammatory drugs (NSAIDs) at least two weeks before the test.
All the patients enrolled in the study were subjected to full history and examination and laboratory investigations: (CBC, Serum Ferritin, Serum Iron, TIBC, Tsat, CRP, serum urea and serum creatinine).

\section{Echocardiographic measurements:}

All patients were examined in detail using standard two-dimensional, pulsewave Doppler, and M-mode echocardiographic methods. Echocardiographic measurements are performed in the left lateral decubitus position according to the recommendations of the American Echocardiography Society. The study was conducted using an ATL HDI 5000 colored echocardiographic machine (Philips IE 33 Colored Echocardiographic Machine, USA) with TDI software incorporated in the device using 2.5$3.5 \mathrm{MHz}$ transducer. The $\mathrm{LV}$ ejection fraction (LV-EF) was calculated automatically according to the modified Simpson method using the software on the echocardiography device. Left ventricular (LV) mass was determined using the method described by (Devereux et al., 1986), and the LV mass index (LVMI) was calculated by dividing LV mass by body surface area. LV hypertrophy (LVH) was defined as a LVMI > $131 \mathrm{~g} / \mathrm{m} 2$ for men and > $100 \mathrm{~g} / \mathrm{m} 2$ for women. LV systolic function was estimated by the LV ejection fraction (LVEF) using a modified biplane Simpson's method from the apical two-chamber and four-chamber views. Left ventricular dimensions (LVEDD, LVESD) were also measured at the end of both the diastolic and systolic phases.

Data were studied utilizing a Statistical package for the Social Science (SPSS) 
version 18.0. Quantitative data were evinced as mean \pm standard deviation $(\mathrm{M} \pm \mathrm{SD})$ while qualitative data were evinced as frequency and percentage
No(\%). Chi-square test: was utilized in comparison of non-parametric data. Pvalues were established statistically significant at $\mathrm{P}<0.05$.

\section{RESULTS}

No statistical significant difference (pvalue $>0.05$ ) was found between studied groups as regard age, sex, Weight and smoking A total of $60 \mathrm{HD}$ patients were included, Patients were classified into two groups according to serum ferritin into 30 patients with serum Ferritin $<800 \mathrm{ng} / \mathrm{ml}$ (Group A) and 30 patients with serum Ferritin $\geq 800 \mathrm{ng} / \mathrm{ml}$ (Group B). The baseline demographic, clinical, and laboratory characteristics was in Table (1, 2).

Among Group A, the mean age was $47.6 \pm 12.3$ years, 19 were males $(63.3 \%)$.
The mean weight was $76.7 \pm 15.5 \mathrm{~kg} / \mathrm{m}^{2}$, 7 patients were smokers $(23.3 \%), 10$ patients were diabetic (33.3\%), 12 patients were hypertensive $(40 \%), 4$ patients with chronic liver disease (13.3\%), and 5 patients with cardiac disease (16.7\%). Among group B, the mean age was 46.4 \pm 10.2 years and 23 were males $(76.7 \%)$. The mean weight was $78.6 \pm 15.5 \mathrm{~kg} / \mathrm{m}^{2}$, 11 patients were smokers $(36.7 \%), 15$ patients were diabetic (50\%), 15 patients were hypertensive $(50 \%), 3$ patients with chronic liver disease (10\%), and 6 patients with cardiac disease $(20 \%)$ (Table 1).

Table (1): Description of demographic data in studied groups, Comparison between studied groups as regard co-morbid conditions

\begin{tabular}{|c|c|c|c|c|c|c|}
\hline \multicolumn{2}{|c|}{$\begin{array}{ll}\text { Parameters } & \text { Groups } \\
\end{array}$} & \multicolumn{2}{|c|}{$\begin{array}{l}\text { Low group } \\
(\mathrm{N}=30)\end{array}$} & \multicolumn{2}{|c|}{$\begin{array}{l}\text { High group } \\
(\mathrm{N}=30)\end{array}$} & P-value \\
\hline \multirow{2}{*}{ Age (years) } & Mean & \multicolumn{2}{|c|}{47.6} & \multicolumn{2}{|c|}{46.4} & \multirow{2}{*}{0.676} \\
\hline & $\pm \mathrm{SD}$ & & & & & \\
\hline \multirow{2}{*}{ Sex } & Male & 19 & $63.3 \%$ & 23 & $76.7 \%$ & \multirow{2}{*}{0.260} \\
\hline & Female & 11 & $36.7 \%$ & 7 & $23.3 \%$ & \\
\hline \multirow{2}{*}{ Weight (kg) } & Mean & \multicolumn{2}{|c|}{76.7} & \multicolumn{2}{|c|}{78.6} & \multirow{2}{*}{0.637} \\
\hline & $\pm \mathrm{SD}$ & & & & & \\
\hline \multirow{2}{*}{ Smoking } & Non & 23 & $76.7 \%$ & 19 & $63.3 \%$ & \multirow{2}{*}{0.260} \\
\hline & Smoker & 7 & $23.3 \%$ & 11 & $36.7 \%$ & \\
\hline & & \multicolumn{2}{|c|}{$\begin{array}{l}\text { Low group } \\
(\mathrm{N}=30)\end{array}$} & \multicolumn{2}{|c|}{$\begin{array}{l}\text { High group } \\
(\mathrm{N}=30)\end{array}$} & P-value \\
\hline \multirow{2}{*}{ HTN } & No & 18 & $60 \%$ & 15 & $50 \%$ & \multirow{2}{*}{0.436} \\
\hline & Yes & 12 & $40 \%$ & 15 & $50 \%$ & \\
\hline \multirow{2}{*}{$\mathrm{DM}$} & No & 20 & $66.7 \%$ & 15 & $50 \%$ & \multirow{2}{*}{0.190} \\
\hline & Yes & 10 & $33.3 \%$ & 15 & $50 \%$ & \\
\hline \multirow{2}{*}{ Liver disease } & No & 26 & $86.7 \%$ & 27 & $90 \%$ & \multirow{2}{*}{0.688} \\
\hline & Yes & 4 & $13.3 \%$ & 3 & $10 \%$ & \\
\hline \multirow{2}{*}{$\begin{array}{l}\text { Cardiac } \\
\text { disease }\end{array}$} & No & 25 & $83.3 \%$ & 24 & $80 \%$ & \multirow{2}{*}{0.739} \\
\hline & Yes & 5 & $16.7 \%$ & 6 & $20 \%$ & \\
\hline
\end{tabular}


No statistical significant difference between studied groups as regard laboratory data except CRP There are no significant differences between the two groups regarding to baseline characteristic and demographic data of both groups of patients, except CRP, which is higher in group B compared with group A ( $\mathrm{p}$-value $<0.05)$. This means that there were significant positive correlations between serum levels of ferritin and CRP (Table 2).

Table (2): Comparison between studied groups as regard laboratory data

\begin{tabular}{|c|c|c|c|c|}
\hline \multicolumn{2}{|c|}{$\begin{array}{ll}\text { Parameters } & \text { Groups } \\
\end{array}$} & $\begin{array}{l}\text { Low group } \\
(\mathrm{N}=30)\end{array}$ & $\begin{array}{l}\text { High group } \\
(\mathrm{N}=30)\end{array}$ & P-value \\
\hline \multirow{2}{*}{ Urea (mg/dl) } & Median & 129.5 & 114.5 & \multirow{2}{*}{0.117} \\
\hline & IQR & $98.5-180$ & $105-129.8$ & \\
\hline \multirow{2}{*}{ Creat (mg/dl) } & Median & 9.8 & 8.7 & \multirow{2}{*}{0.076} \\
\hline & IQR & $8.2-12.3$ & $7.3-10.3$ & \\
\hline \multirow{2}{*}{$\mathrm{Hb}(\mathrm{g} / \mathrm{dl})$} & Median & 9.4 & 9.3 & \multirow{2}{*}{0.912} \\
\hline & IQR & $8.9-10.2$ & $8.6-10.2$ & \\
\hline \multirow{2}{*}{ PLTs $\left(\mathrm{x} 10^{3} / \mathrm{ul}\right)$} & Median & 252.5 & 256.5 & \multirow{2}{*}{0.982} \\
\hline & IQR & $154.8-338$ & $148-345$ & \\
\hline \multirow{2}{*}{$\begin{array}{l}\text { WBCs } \\
\left(\mathrm{x} 10^{3} / \mathrm{ul}\right)\end{array}$} & Median & 7.25 & 7.7 & \multirow{2}{*}{0.455} \\
\hline & IQR & $4.6-9.9$ & $5.8-9.6$ & \\
\hline \multirow{2}{*}{$\mathrm{CRP}(\mathrm{mg} / \mathrm{L})$} & Median & 3.6 & 4.7 & \multirow{2}{*}{0.007} \\
\hline & IQR & $3.2-4.5$ & $3.8-6.6$ & \\
\hline \multirow{2}{*}{$\begin{array}{l}\text { Calcium } \\
(\mathrm{mg} / \mathrm{dl})\end{array}$} & Median & 8.6 & 8.7 & \multirow{2}{*}{0.767} \\
\hline & IQR & $7.7-9.6$ & $8.2-9.5$ & \\
\hline
\end{tabular}

There were statistically significant differences between studied groups as regard echocardiographic findings $\mathrm{We}$ found statistically significant difference (p-value $<0.05$ ) between studied groups as regard LVESD \& LVM/Ht2.7, and highly statistical significant difference (pvalue $<0.001$ ) between studied groups as regard LVEDD \& LVMI. The LVEDD, LVMI, LVESD \& LVM/Ht2.7 values were significantly higher in group B compared with group A (Table 3).

Table(3): Comparison between studied groups as regard echocardiographic findings

\begin{tabular}{|c|c|c|c|c|}
\hline \multicolumn{2}{|c|}{$\begin{array}{ll}\text { Parameters } & \text { Groups } \\
\end{array}$} & $\begin{array}{l}\text { Low group } \\
(\mathrm{N}=30)\end{array}$ & $\begin{array}{l}\text { High group } \\
(\mathrm{N}=30)\end{array}$ & P-value \\
\hline \multirow{2}{*}{ LVEDD } & Median & 5.2 & 6 & \multirow{2}{*}{$<0.001$} \\
\hline & IQR & $4.7-5.4$ & $5.2-6.3$ & \\
\hline \multirow{2}{*}{ LVESD } & Mean & 3.3 & 3.5 & \multirow{2}{*}{0.008} \\
\hline & $\pm \mathrm{SD}$ & $3.1-3.4$ & $3.2-3.8$ & \\
\hline \multirow{2}{*}{ LVMI } & Mean & 145.5 & 182.5 & \multirow{2}{*}{$<0.001$} \\
\hline & $\pm \mathrm{SD}$ & $127.5-167.5$ & $165.8-200.3$ & \\
\hline \multirow{2}{*}{$\begin{array}{l}\mathrm{LVM} / \mathrm{Ht} \\
2.7 \\
\end{array}$} & Mean & 69.5 & 80.5 & \multirow{2}{*}{0.01} \\
\hline & $\pm \mathrm{SD}$ & $59.8-81.8$ & $70.3-88.3$ & \\
\hline \multirow{2}{*}{ EF } & Mean & 69.8 & 70.3 & \multirow{2}{*}{0.190} \\
\hline & $\pm \mathrm{SD}$ & $69.3-70.5$ & $69.2-71.7$ & \\
\hline
\end{tabular}




\section{DISCUSSION}

In the current study, we found a statistically significant difference between studied groups as regard LVESD and LVM/Ht2.7, and statistical significant difference between studied groups as regard LVEDD and LVMI. The LVEDD, LVMI, LVESD and LVM/Ht2.7 values were significantly higher in group B compared with group A. LVM, LVMI and LVM/Ht2.7 were significantly correlated to ferritin.

The results of current study were supported by Eldeeb et al. (2018) who showed that patients with serum ferritin level $\geq 800 \mathrm{ng} / \mathrm{ml}$ were significantly higher LVM, LVMI and LVM/Ht.

$\mathrm{Fu}$ et al. (2020) performed a retrospective, observational, cohort study which showed that the underlying cardiovascular mortality was higher among PD patients with serum ferritin values $\geq 100 \mu \mathrm{g} / \mathrm{L}$ than among those with ferritin levels $<100 \mu \mathrm{g} / \mathrm{L}$. A multivariate Cox regression analysis revealed that an increased serum ferritin level was independently associated with a higher risk of cardiovascular mortality in PD patients after adjusting for relevant confounding factors.

Moreover, Kuragano et al. (2014) performed a prospective, observational, multicenter study of 1086 Japanese HD patients and found that hyperferritinemia, defined as serum ferritin $>100 \mu \mathrm{g} / \mathrm{L}$, is a risk factor for cardiovascular disease, hospitalization and death.

Son et al. (2019) retrospectively collected the data of outpatients on maintenance hemodialysis for 5 years. They showed that ferritin levels > 100 $\mathrm{ng} / \mathrm{mL}$ is associated with increased rates of cardiovascular events.

Our results showed that CRP levels were higher in the high Ferritin group compared with low Ferritin group with statistically significant difference between them.

This results supported by Elmenyawi et al. (2017) who reported significant positive correlations between serum levels of ferritin and CRP.

Abd El-Hafeez et al. (2019) studied the association between inflammatory markers in hemodialysis patients. They observed statistically significant positive correlation between hs-CRP and serum Ferritin.

\section{CONCLUSION}

High serum ferritin level ( $\geq 800 \mu \mathrm{g} / \mathrm{L})$ was positively associated with Left ventricular hypertrophy in maintenance hemodialysis patients.

\section{Conflict of Interest:}

The authors of the study have no conflict of interest related to this publication.

\section{REFERENCES}

1. Abdel-Hady H, Khamis S, Salah Y, Elbarbary H, Zahir E and Elmahmoudy A (2013): Fibroblast growth factor 23 as a risk factor of left ventricular hypertrophy and vascular calcification in predialysis chronic kidney disease patients. Menoufia Med J, 26:717.

2. Abd El-Hafeez, A., Ahmed, H., Al-Alashkar, A. M., Mohamed, N. M. A and Zoheir, M. I. K. (2019): Association between Neutrophil to Lymphocyte Ratio and Inflammatory Markers in Hemodialysis Patients. The Egyptian Journal of Hospital Medicine, 77(5), 5681-5689. 
3. Eldeeb, A., El-Adawy, A. H., Yousef, E., Ibrahim, A., Mahmoud, M. A., Shakour, H. A and Omran, M. (2018): Relation between Serum Ferritin Level and the Left Ventricular Mass Index (LVMI) in Maintenance Hemodialysis Patients. Cardiology and Cardiovascular Research, 2(4):98.

4. Elmenyawi, A. A., Hassan, A., Said, S. A and Sawar, S. (2017): Relationship between hepcidin, ferritin and C-reactive protein in hemodialysis patients. The Egyptian Journal of Hospital Medicine, 69(2): 1786-1793.

5. Fu, S., Chen, J., Liu, B., Liang, P., Zeng, Y., Feng, M (2020): Systemic inflammation modulates the ability of serum ferritin to predict all-cause and cardiovascular mortality in peritoneal dialysis patients. BMC Nephrology, 21(1): 1-9.

6. Khanna V, Karjodkar F, Robbins S, Behl M, Arya $S$ and Tripathi A. (2017): Estimation of serum ferritin level in potentially malignant disorders, oral squamous cell carcinoma, and treated cases of oral squamous cell carcinoma. J Cancer Res Therj, 13(3):550 5 .

7. Kuragano, T., Matsumura, O., Matsuda, A., Hara, T., Kiyomoto, H., Murata, T., ... \& Fukatsu, A. (2014): Association between hemoglobin variability, serum ferritin levels, and adverse events/mortality in maintenance hemodialysis patients. Kidney International, 86(4), 845-854.

8. Lien CT, Lin KC, Tsai YF, Yu LK, Huang LH and Chen CA (2015): Serum ferritin is associated with progression of peripheral arterial disease in hemodialysis patients. Clin Exp Nephrology, 19(5):947-52.

9. Monfared, A., Salari, A., Kazemnezhad, E., Lebadi, M., Khosravi, M., Mehrjardi, N. K., ... and Amini, N. (2013): Association of left ventricular hypertrophy with high-sensitive Creactive protein in hemodialysis patients. International urology and nephrology, 45(6), 1679-1686.

10.Son, D. Y., Ono, L. K. and Qi, Y. (2019): Association between hemoglobin variability, serum ferritin levels, and adverse events/mortality in maintenance hemodialysis patients. Kidney international, 86(4), 845-854.

11. Xu, Y., Chen, Y., Li, D., Li, J., Liu, X., Cui, C., and Yu, C. (2013): Hypertension, fluid overload and micro inflammation are associated with left ventricular hypertrophy in maintenance hemodialysis patients. Renal Failure, 35(9): 1204-1209. 


\section{العلاقة بين مستوي الفيريتين في اللدم ووظائف البطينى الأيسر في مرضي الاستصفاء اللدموي ولئي}

احمد لطفي احمد*، فوزي حامد حسن*، السيد محمد راشد*، محمد أحمد مسعد***،

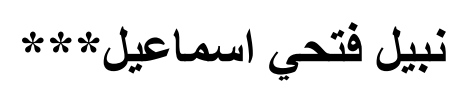
أقسام الباطنه والقلب والباثولوجيا الاكلينيكية، كلية الطب، جامعة الازهر

E-mail: lotfiahmed272@gmail.com

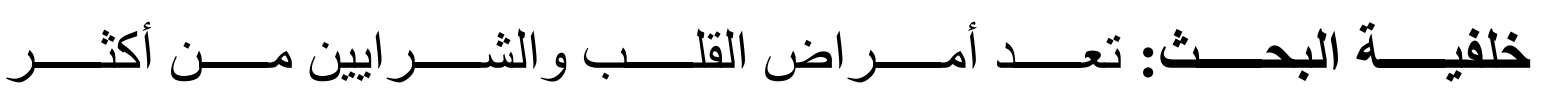

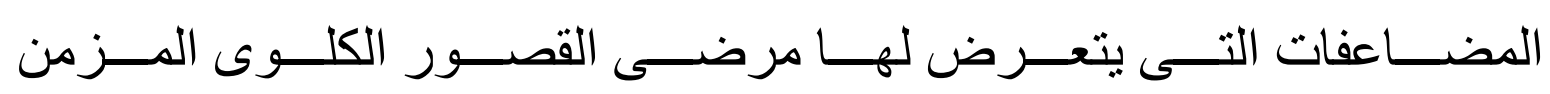

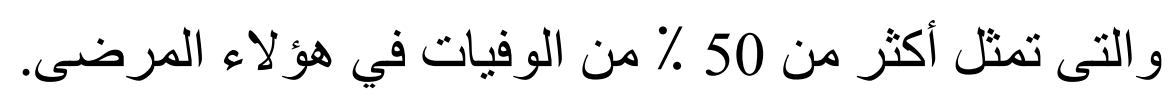

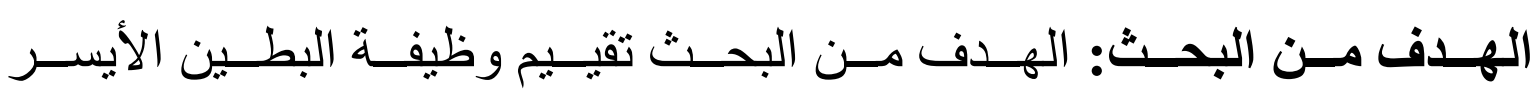

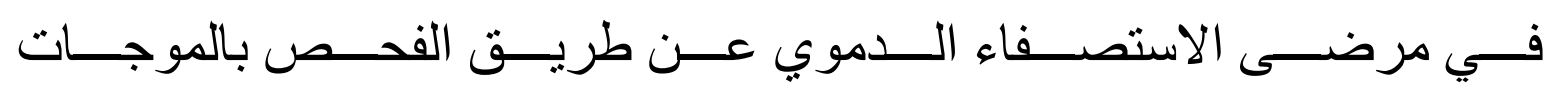

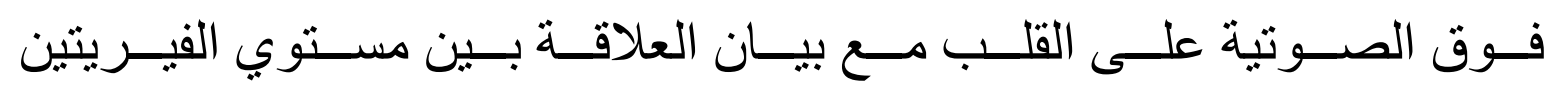

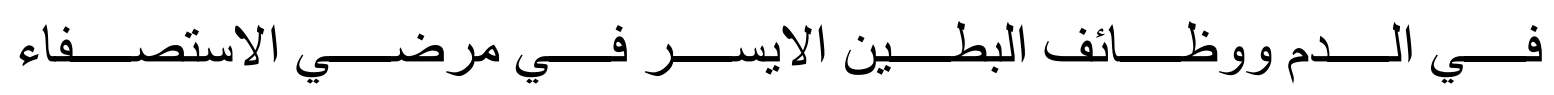
الدموي.

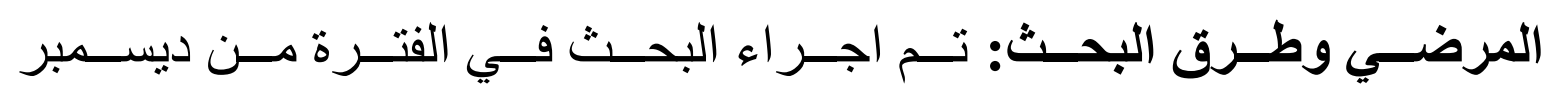

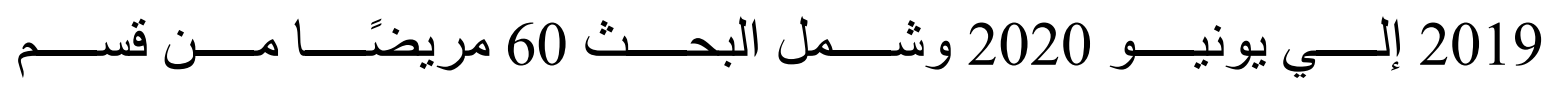

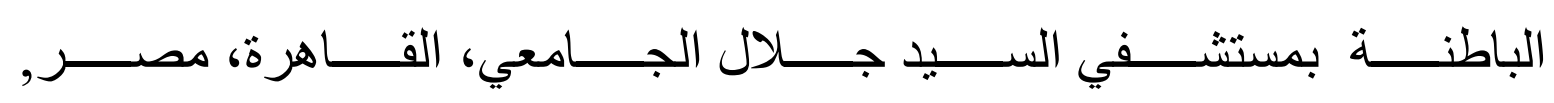

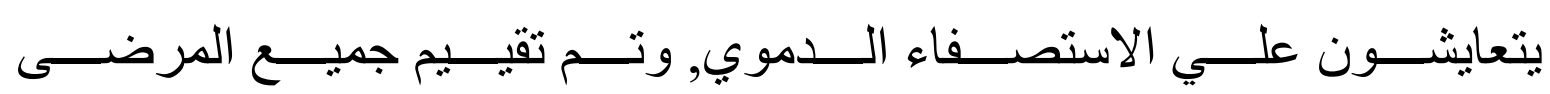

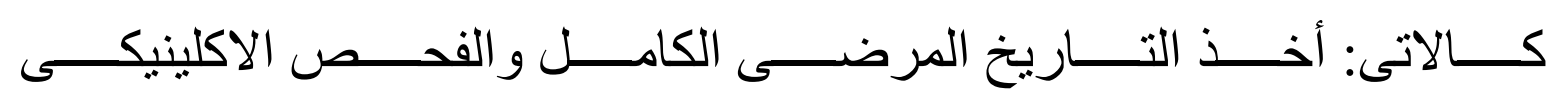

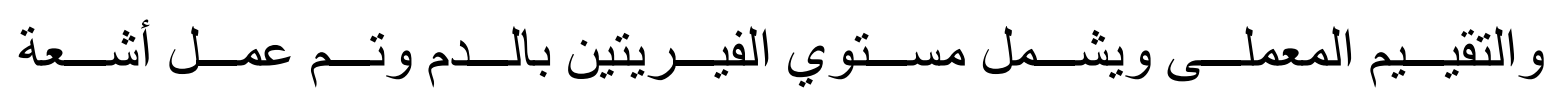

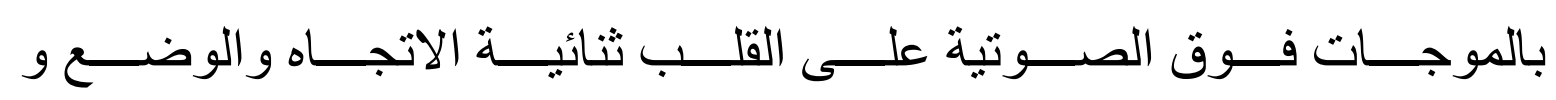

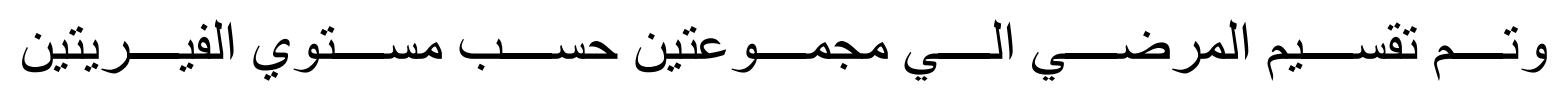




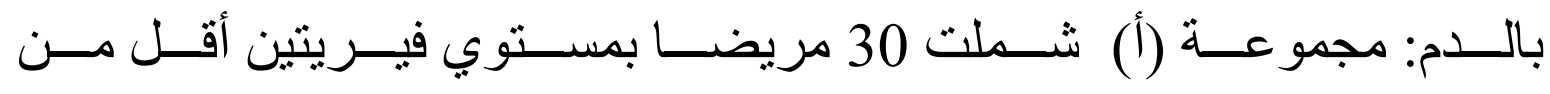

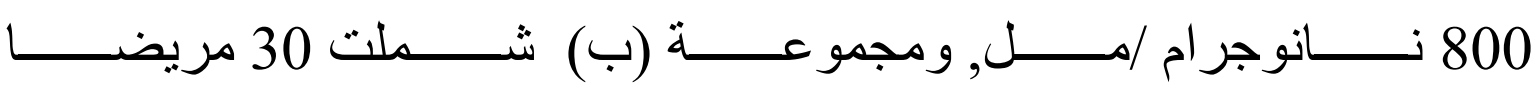
بمستوي فيريتين أكثر من 800 نانوجر ام/مل.

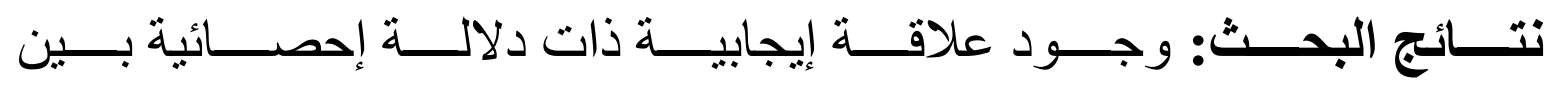

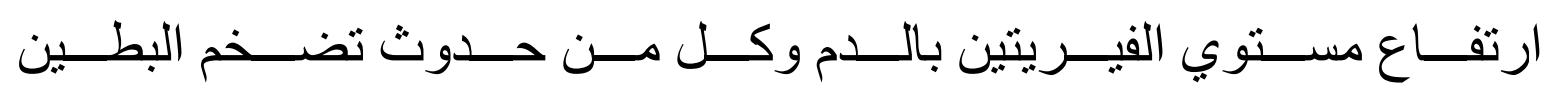
الأيسروزيادة مستوى بروتين سى التفاعلى.

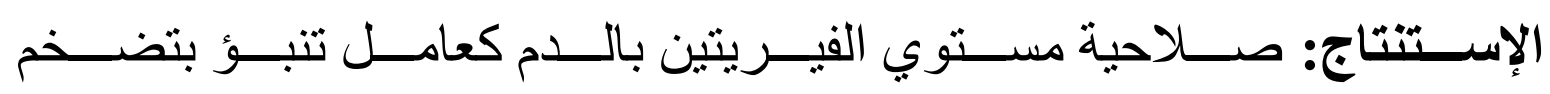

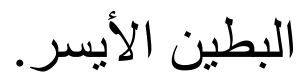

الكلمـــات الألـــة: وظـــائف البطـــين الأيســـر ، مســـتوى الفيــرتين فــى

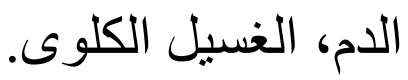

